\title{
Alpha Thalassemia
}

National Cancer Institute

\section{Source}

National Cancer Institute. Alpha Thalassemia. NCI Thesaurus. Code C34368.

A genetic hematologic disorder characterized by partial or complete absence of the alpha globin chains of the heme molecule. 